\title{
Radar micro-Doppler for loudspeaker analysis: an industrial process application
}

\author{
A. Izzo*, L. Ausiello ${ }^{\dagger}$, C. Clemente*, J.J. Soraghan* \\ ${ }^{*}$ University of Strathclyde, Centre for Signal and Image Processing (CeSIP), EEE, 204, George Street, G1 1XW, Glasgow, UK. \\ E-mail: alessio.izzo, carmine.clemente, j.soraghan, -@strath.ac.uk \\ ${ }^{\dagger}$ HouseYellow Acoustic and Audio Consulting. E-mail:ludwig.ausiello@gmail.com
}

\begin{abstract}
In the last decades a strong interest has developed on radar micro-Doppler analysis targeting applications in a number of sectors including defence, bio-medical and automotive. This paper investigates for the first time the use of radar microDoppler for loudspeaker analysis. This approach offers the potential benefits to characterize the mechanical motion of a loudspeaker and to identify defects and design issues. Compared with acoustic based approaches, the use of radar information allows reliable measurements in an acoustically impaired environment. In addition, when compared with a laser vibrometric approach, the use of micro-Doppler reduces the number of measurements required and provides direct access to the information of the metallic components of the loudspeaker. In the paper experimental results and analysis of the micro-Doppler signatures of loudspeakers using low cost radar are presented.
\end{abstract}

Keywords: Radar, micro-Doppler, micro-Doppler analysis, loudspeaker analysis, industrial processes.

\section{Introduction}

The interest for micro-Doppler analysis has grown in the past decades, reaching a plethora of sectors and applications [1, 2]. In defence application, the micro-Doppler signature has been used to detect and classify targets. In [3], it has been used for automatic helicopters classification. In [4] the method is exploited to discriminate birds and small unmanned aerial vehicles (UAVs) with emphasis on micro-Doppler that can be extracted from spectrograms and cepstrograms. In [5] a microDoppler based algorithm has been presented for ballistic missile classification. In the bio-medical field, the micro-Doppler signature is used to estimate the total human energy expenditure for walking and running activities [6]. In automotive, micro-Doppler analysis has been used to classify pedestrian activity for Automatic Drive Assistant Systems (ADAS) [7]. The interest in micro-Doppler suggests that this technology is reliable and ready, and that it is worthwhile investigating it in further applications domains.

This paper introduces a novel approach to loudspeaker analysis based on radar micro-Doppler signatures. Our approach is motivated by the potential advantages that a radar based approach could introduce over acoustic and laser based ones. The former approach is widely spread in R\&D and manufacturing of acoustic transducers and consumer products (e.g. loudspeakers or audio products), and the latter is more frequently used in advanced markets like automotive audio components and systems. However, both have technical and practical limitations that are discussed as follows.

The effectiveness of acoustic End-Of-Line tests (EOL) or acoustic measurements is limited by the surrounding environment; it normally requires specifically designed insulated booths or silent areas for the signal-to-noise ratio of audio data to be meaningful. In cases where laser-based scanner vibrometer systems (Scanning Vibrometer System (SCN) [9]) would be preferable to the traditional acoustic one, we have to highlight two serious downsides: the first is the necessity of very large sets of measurements (up to almost 3000 points) to fully characterize a loudspeaker and its non linearities, thus being a serious time consuming activity, the second is the limitation due to the presence of any physical obstacle in the line of sight between the laser source and the membrane (or acoustic source) under test [10, 11]. In both the applications the use of micro-Doppler could be sensibly cost effective. Despite the traditional acoustic measurement, it will ensure independency from environmental acoustic noise, allowing its use in end of production lines. Despite the SCN system, the microDoppler has the ability to cope with visual occlusion due to plastic parts and the capability of separation metallic components of a loudspeaker from non metallic ones through the use of the back-scattering intensity.

In this paper the most common embodiment of electrodynamic transducer will be introduced in section 2 , followed, in section 3, by a simple model for micro-Doppler signature of a driver in a loudspeaker. Section 4 presents results of experimental acquisitions compared with simulated results validating the expected micro-Doppler modulations. Finally, Section 5 will present the conclusions and the future developments.

\section{Loudspeaker kinematic}

An electro-dynamic or moving-coil loudspeaker is an electromagnetic actuator aimed to convert electrical signals into 
sounds. There is a plethora of types of transducers [13], each one relying on a different working principle to transmit energy to air. Almost all of them need a vibrating surface, namely the diaphragm, to transmit motion to air molecules. Thus the sound is radiated. Focusing on the direct-radiator type, a section of this kind of speaker is shown in Figure 1 .

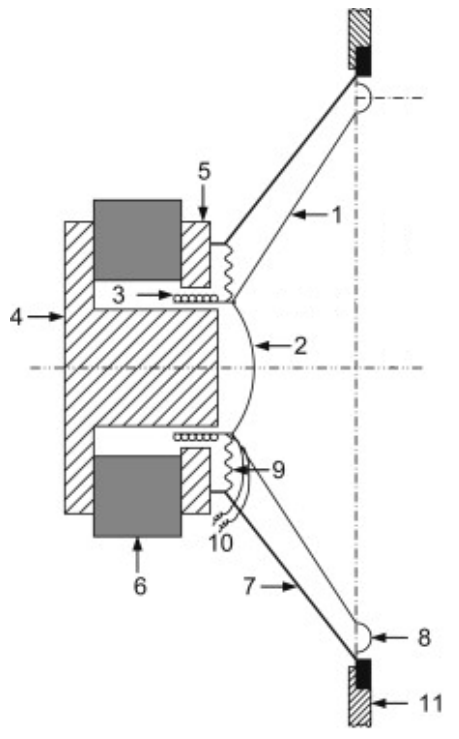

1.Cone

2.Dust cap

3.Voice coil

4.Pole piece

5.Pole plate

6.Permanent magnet

7.Basket

8.Surround

9.Spider

10.Electrical connection

11.Infinitely large baffle

Figure 1: Cross sectional sketch of a direct-radiator loudspeaker.

The diaphragm, often called cone, is made from a suitably light and stiff material; most of its stiffness comes from its profile, which could be either a straight line (a real cone) or a curved one. In the center, a dust cap is placed which guards against metallic dust fouling into the magnetic gap and prevents sound from the back of the diaphragm leaking through to the outside world. Attached to the top of the cone is a cylinder called coilformer, around which the coil is wrapped. The coil is located in the gap of a magnetic path, comprising a pole piece and pole plate, where the magnetic flux is produced by a permanent magnet, which is held in place by a basket structure. The diaphragm is supported at the rim and near the voice coil by a surround and spider, respectively, so that it is free to move only in an axial direction 1 .

When an audio signal is applied to the voice coil, the resulting current creates a magnetomotive force which interacts with the air-gap flux of the permanent magnet and causes a translatory movement of the voice coil and, hence, of the cone to which it is attached. The movement of the cone in turn displaces the air molecules at its surface thus producing sound waves. The strength and therefore its velocity, by which the cone moves and pushes the surrounding air produces the loudness of the sound [13].

\footnotetext{
${ }^{1}$ In general, sound from the back of the cone exits through holes in the basket, while sound from the back of the dust cap leaks through the magnetic gap and spider, which often presents holes or porosity, before exiting through the basket.
}

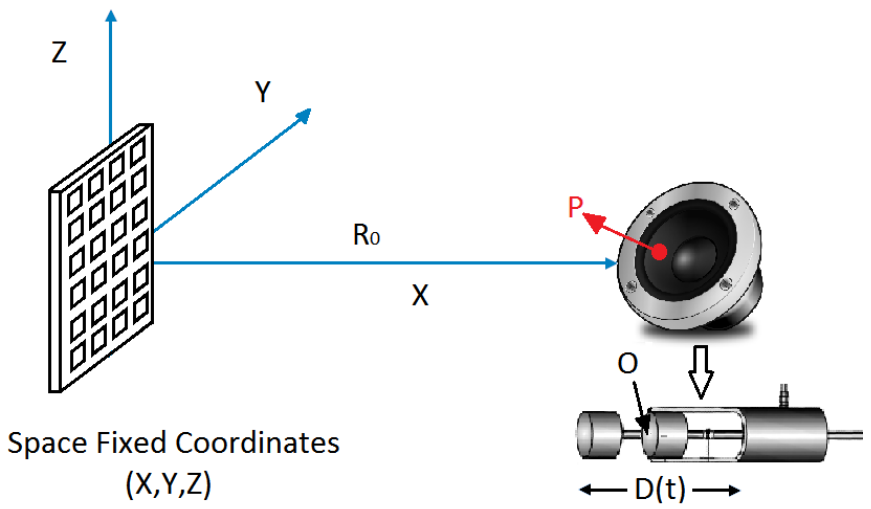

Figure 2: Geometry for the radar and generic vibrating point: the motion of a speaker can be described as rigid body motion having a piston mode when the input to the loudspeaker is a signal with frequency range up to $1 k H z$.

All low frequency models ( below $1 \mathrm{kHz}$ ) of dynamic loudspeakers generally assume that the drivers operate in what is referred to as the piston mode. This generally means that in this frequency range the driver cone behaves as a rigid body. Measurements show that real drivers are never rigid though, and that it is practically impossible to make a perfect piston but for a small frequency bandwidth, which is related to the physical dimension of the diaphragm [10, 11, 14]. This behaviour, together with the need of an efficient transduction, are the reasons for the use of several different transducers (woofers, midranges and tweeters) having specific dimensions and shapes to properly cover the whole audible spectrum.

Having briefly introduced some of the aspects of the electrodynamic transducer motion, in our experimental analysis we will firstly focus on the rigid body motion of the loudspeaker at low frequencies in order to detect and confirm its behaviour through micro-Doppler signature.

\section{Micro-Doppler Analysis}

The mathematics of radar micro-Doppler effect can be derived from introducing micro-motion to the conventional Doppler analysis. In this section the basics of the micro-Doppler will be introduced. This is crucial for understanding and correctly interpreting the micro-Doppler phenomena in more complex and realistic cases. In Figure 2 the geometry used to analyse the micro-Doppler induced by a vibrating target is shown [1, 2].

An arbitrary point of the cone located in $P$ vibrates with sinusoidal frequency $f_{v}$ and displacement $D_{v}$, thus having a displacement function of the kind:

$$
D(t)=D_{v} \sin \left(2 \pi f_{v} t\right)
$$

while assuming the radar being in Line Of Sight (LOS) with the speaker [1, 2]. Letting $R_{0}$ be the distance between the 
radar and the speaker initial position $O$, then the range function varies with time due to the speaker micro-motion:

$$
R(t)=R_{0}+D(t) .
$$

The radar received signal becomes

$$
s(t)=\rho \exp \left\{j\left[2 \pi f_{0} t+4 \pi \frac{R(t)}{\lambda}\right]\right\}
$$

where $\rho$ is the backscattering coefficient, $f_{0}$ is the carrier frequency and $\lambda$ is the carrier wavelength. Substituting $R(t)$ in (3), the received signal can be expressed as:

$$
s(t)=\rho \exp \left\{j \frac{4 \pi R_{0}}{\lambda}\right\} \exp \left\{j 2 \pi f_{0} t+\frac{4 \pi D_{v}}{\lambda} \sin \left(w_{v} t\right)\right\}
$$

where $w_{v}=2 \pi f_{v}$. From (4), the derivative of the second phase term leads to the expression of the micro-Doppler shift:

$$
f_{m D}(t)=\frac{2 w_{v} D_{v}}{\lambda} \cos \left(w_{v} t\right)
$$

From this simple set of equations describing the microDoppler signature we can deduct the capability to retrieve information about the behaviour, anomalies and failures of a loudspeaker from the returned signal.

\section{Simulated and Real measurement analysis}

In this section real data acquisitions are analysed and compared with simulated one following the model described in Section 3

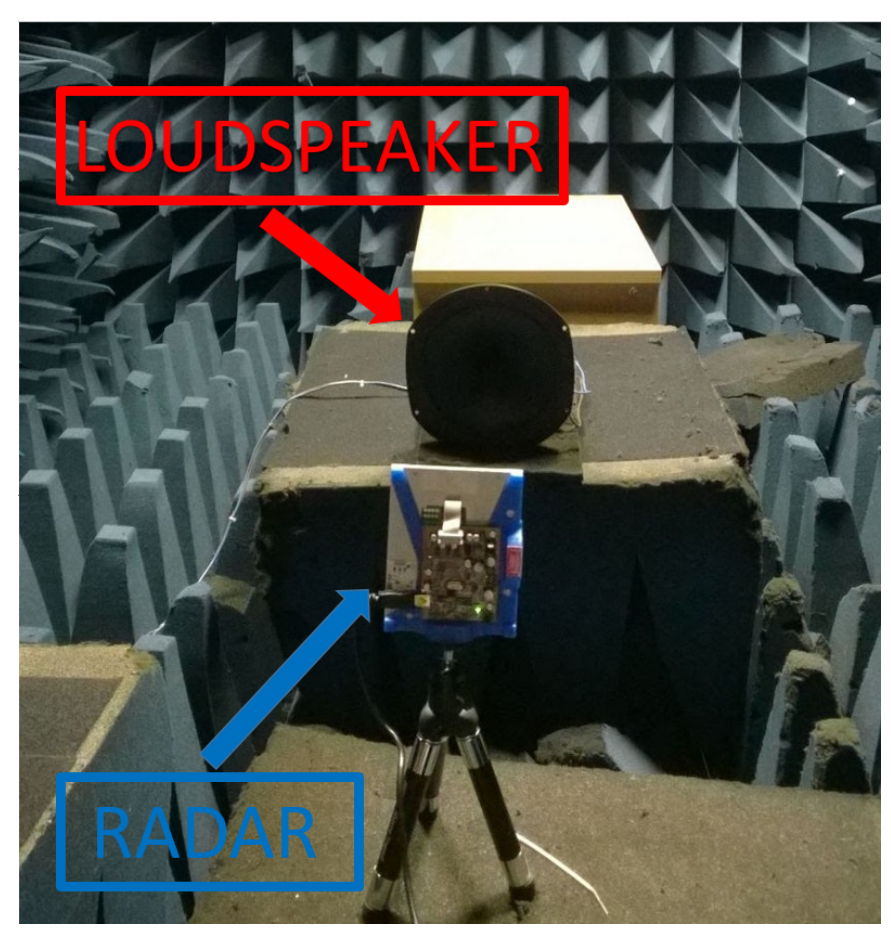

Figure 3: Experiment setup.
Both the simulated and real data are generated using an acoustic input to the loudspeaker at $f_{v}=125 \mathrm{~Hz}$ with amplitude $-6 \mathrm{~dB}$ for the standard "Loudness units relative to Full Scale" (LUFS). In order to simulate a received radar signal, a diameter of $16.5 \mathrm{~cm}$ (which is a typical dimension for a loudspeaker operating in this frequency range) has been considered. The backscattering coefficient $\rho$ [2] coming from the only vibrating metallic component, namely the voice coil, is calculated as:

$$
\rho=\frac{4 \pi^{3} r^{4}}{\lambda^{2}}
$$

with $\lambda$ the radar signal wavelength and $r=1.3 \mathrm{~cm}$ the ray of voice coil. Under this assumption, the received radar signal in baseband has been simulated as:

$$
s(t)=\frac{\rho}{\left(R_{0}+D_{v} \sin \left(2 \pi f_{v} t\right)\right)^{2}} \exp \left(\frac{j 4 \pi D_{v} \sin \left(2 \pi f_{v} t\right)}{\lambda}\right)
$$

The measurements acquisition have been done through a 24 $\mathrm{GHz} \mathrm{CW}$ radar made by WhiteHorse Radar LTD. It has been used to measure the returns from a $16.5 \mathrm{~cm}$ ProSound subwoofer placed $1 \mathrm{~m}$ far away from the radar on the line of sight. The system setup and the speaker under test are shown in Figure 3. The speaker was connected to an amplifier also placed in the anechoic chamber. The input signal to the loudspeaker has been generated by Adobe Audition 3.0, while the received signal is acquired by the radar through Audacity. The data obtained were processed through Matlab R2016a.

A well known technique to extract the micro-Doppler features is the spectrogram [3, 4]. A spectrogram is a timefrequency distribution useful for visualization and analysis of non-stationary signals. It is obtained by taking the squared magnitude of the short-time Fourier transform (STFT) of a discrete signal $s(n)$, written as:

$$
\begin{aligned}
\chi(\nu, k) & =|\operatorname{STFT}\{s(n)\}|^{2}= \\
& =\left|\sum_{n=0}^{N-1}\left(s(n) h(n-k) \exp \frac{-j 2 \pi \nu n}{N}\right)\right|^{2}
\end{aligned}
$$

where $k=0, \ldots, K-1, \nu$ is the normalized frequency and $h$ is a discrete window function of choice [8]. The spectrograms of both simulated and real data are produced using a Hamming window of $29 \mathrm{~ms}$, with an overlap of $90 \%$, and are shown in Figure 4 The behaviour depicted in the Figure 4 b) is in agreement with the model (Figure 4(a)), confirming the speaker is moving in piston mode at this frequency. From the spectrogram the maximum frequency Doppler shift can be evaluated and through it even the displacement information can be extracted. By inverting the equation (5), the maximum value of $D_{v}$ can be obtained. The measured maximum of the microDoppler shift resulted to be $251.5 \mathrm{~Hz}$, leading to an estimated displacement $D_{v}=2 \mathrm{~mm}$. 


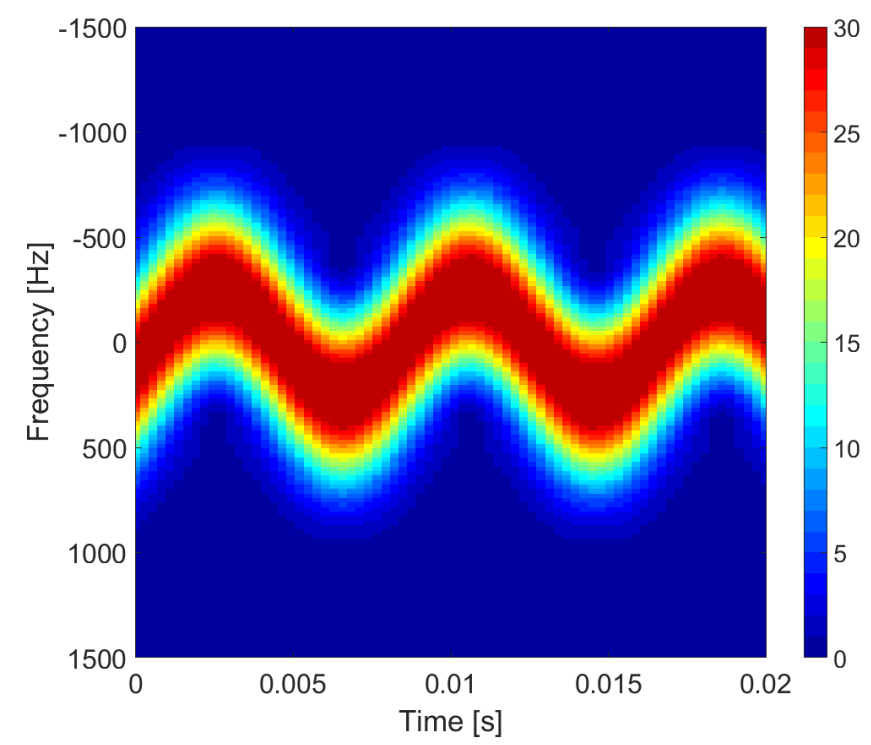

(a) Simulated spectrogram

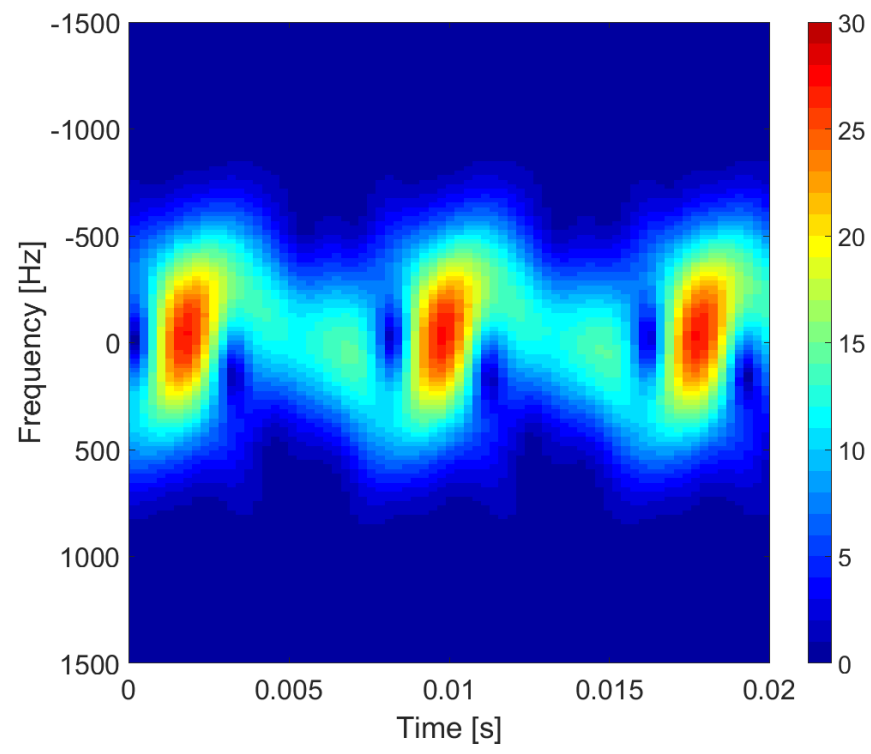

(b) Real spectrogram

Figure 4: Simulated and real spectrogram of radar measurement coming from a loudspeaker with a voice voice ray of $r=1.3 \mathrm{~cm}$.

In Figure 5 the phase of the real and simulated radar signals are reported. It can be appreciated how the phase of the real data matches the simulated one in terms of sinusoidal-like motion. However, discrepancies between the simulated and real data suggests the presence of a non-linear behaviour in the voice coil motion. Comparing the rising and falling front of the measured phase with the corresponding simulated one, it is possible to observe that actual motion does not have a constant acceleration. From the plot it is possible to infer that the voice coil spends more time in the position further away from the radar than the piston model suggests. A possible explanation

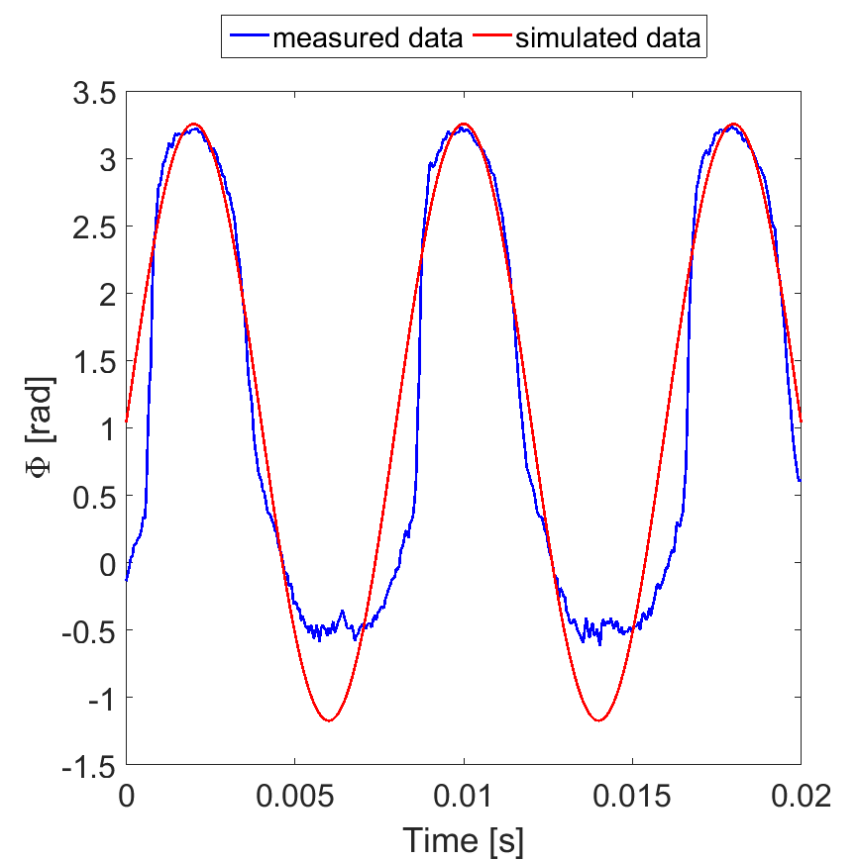

Figure 5: Simulated and real phase of the received radar signal when an input at $125 \mathrm{~Hz}$ and $-6 \mathrm{~dB}$ LUFT is applied to a loudspeaker.

of this phenomenon could be the superposition of two components: one is the non linearity of the elastic force generated by the to mechanical support of the cone, while the other component could be the non linearity of the force generated by the magnetic field times the length of the voice coil immersed in the gap [11]. As soon as the coil does not vibrate anymore, it is pushed back by magnetic field increasing the slope of rising front.

To assess the rate of change in a spectrum, cepstral analysis can be used. It is based on the power cepstrum, which is defined as the power of the inverse Fourier transform of the logarithm of the power spectrum [4, 15]:

$$
\Xi\{s(n)\}=\left|\mathcal{F}^{-1}\left\{\log \left(\mathcal{F}|s(n)|^{2}\right)\right\}\right|^{2}
$$

where the free variable has the dimension of time and has been coined "quefrency". Using equation (9), the periodic pattern of the micro-Doppler can be evaluated through the cepstrum. The simulated and measured data are compared in figure 6 , It is interesting to mark that while the cepstrum based on simulated data was calculated in absence of noise, real measurements presented a noise floor, however it is still clear a peak at the quefrency of $8 \mathrm{~ms}$ in both the real and simulated data. Finally, the same analysis has been performed with an input signal having $f_{v}=31.5 \mathrm{~Hz}$ and three different amplitude values. In Figure 7, the spectrograms of a input signal with amplitudes of $-6 \mathrm{~dB},-12 \mathrm{~dB}$ and $-18 \mathrm{~dB}$ are shown. Referred to the signal with amplitude equal to $-6 \mathrm{~dB}$, the maximum Doppler shift value is $f_{m D_{-6}}=292.5 \mathrm{~Hz}$. By inverting the equation (5), the 


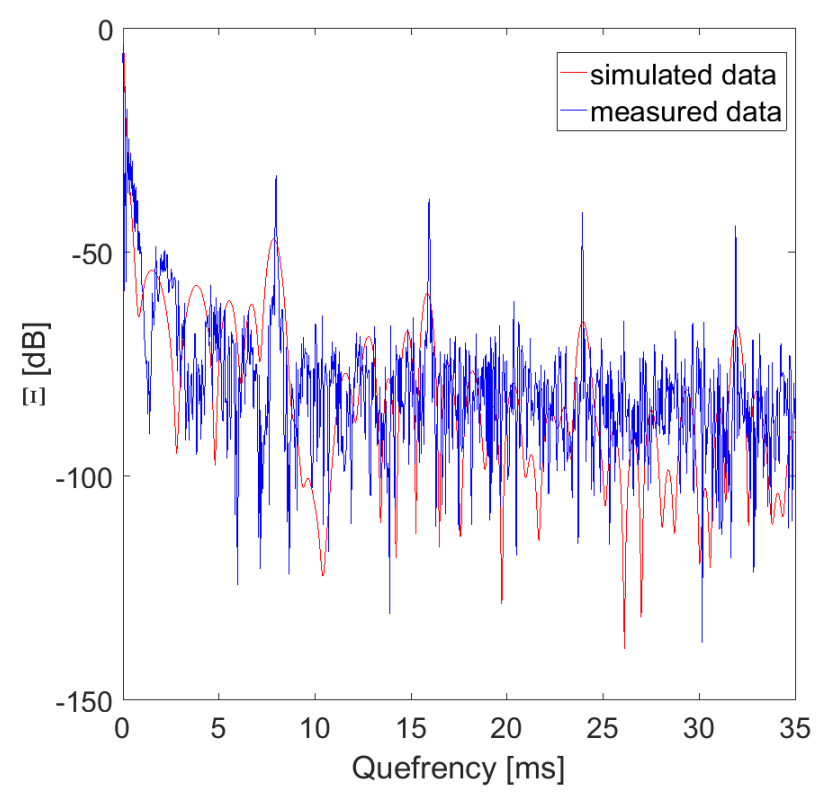

Figure 6: Simulated and real cepstrum of radar measurement coming from a 6.5 inch speaker: the peak at $8 \mathrm{~ms}$ is referred to the micro-Doppler periodicity of a single tone at $125 \mathrm{~Hz}$.

measured displacement value is $D_{v_{-6}}=9.2 \mathrm{~mm}$. As expected, a change of the maximum micro-Doppler shift appears when the input amplitude changes. In particular, when the amplitude is $-12 \mathrm{~dB}$ and $-18 \mathrm{~dB}$, a maximum micro-Doppler shift of $f_{m D_{-12}}=277 \mathrm{~Hz}$ and $f_{m D_{-18}}=192 \mathrm{~Hz}$ is observed leading to a spatial displacement of $D_{v_{-12}}=8.7 \mathrm{~mm}$ and $D_{v_{-18}}=6$ $\mathrm{mm}$ respectively. Nevertheless, from the cepstrum in Figure 8. the frequency of the input signal can be detected looking at the micro-Doppler periodicity. This is important if we want to rely also on this technique as a valid tool to detect anomalies, or vibrational behaviours that differ from the linear/expected ones.

\section{Conclusion}

In this paper a novel approach based on radar micro-Doppler has been introduced for the first time to analyse and measure a loudspeaker. At low frequency, the speaker has been modelled as a piston since all its components are moving in phase. From the received radar signal, spectrogram, phase and cepstrum were compared with the model confirming that the motion and other kinematic characteristics can be detected. In particular, considering the phase component of the received signal, the information of the displacement motion can be extracted, as in the case study presented where a non linear behaviour of the voice coil was highlighted by the radar measurement. From the micro-Doppler signature, non linearity could be identified making this approach a powerful tool for the evaluation of mass produced loudspeakers in acoustically impaired environment. Finally, when the wave length of the sound in air

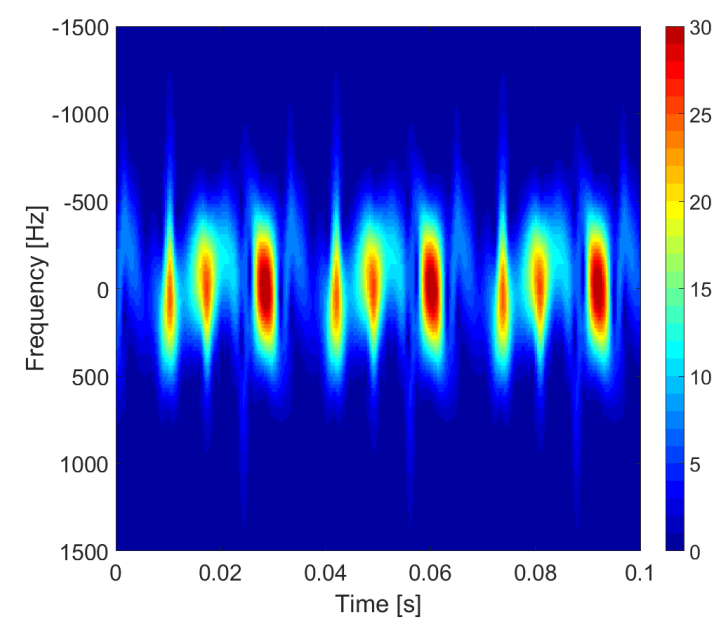

(a) $-6 \mathrm{~dB}$

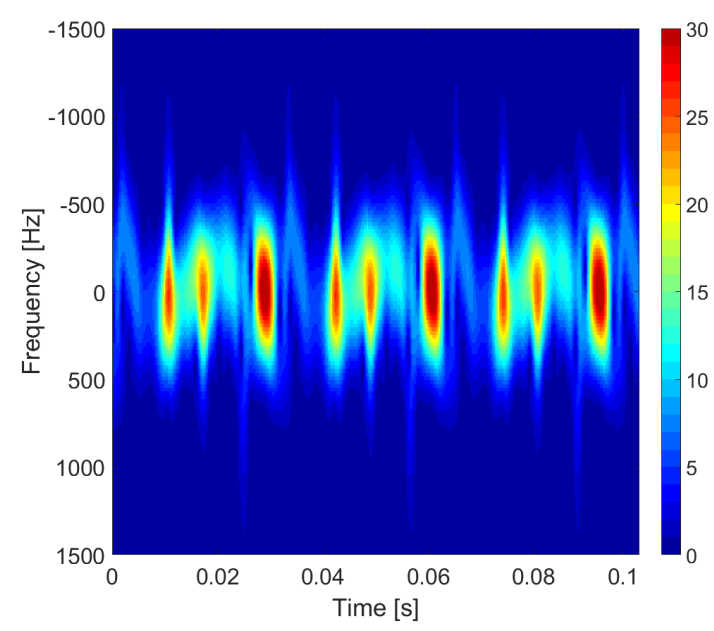

(b) $-12 \mathrm{~dB}$

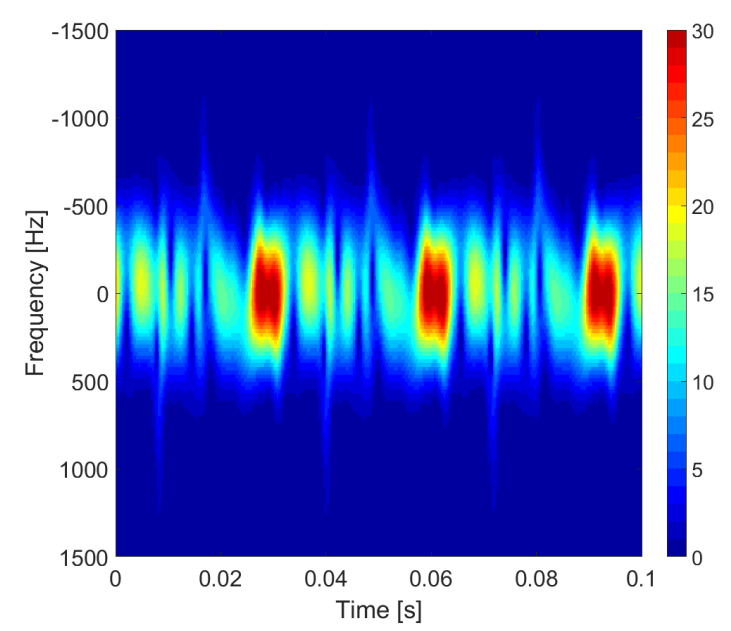

(c) $-18 \mathrm{~dB}$

Figure 7: Real spectrograms of radar measurement with input signal having $f_{v}=31.5 \mathrm{~Hz}$ with amplitude values of $-6 \mathrm{~dB},-12 \mathrm{~dB}$ and $-18 \mathrm{~dB}$. 


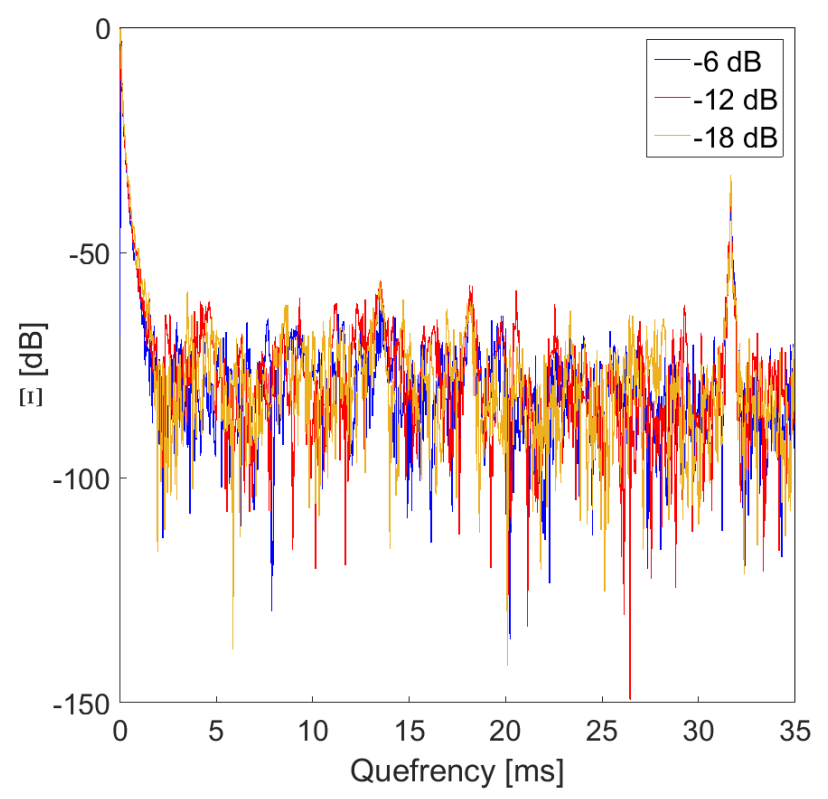

Figure 8: Real cepstrum of radar measurement coming from a 6.5 inch speaker: the peak at $33 \mathrm{~ms}$ is referred to the micro-Doppler periodicity of a input signal having $f_{v}=31.5 \mathrm{~Hz}$.

is comparable to or less than twice the cone diameter (above $1 \mathrm{kHz}$ ), the driver cone does not behave as rigid body and should be modelled as a flexible system. The vibrations travel transversally along the cone surface generating rocking modes which produce non linear or undesired output. For this reason, it is anticipated that additional modelling and signal processing tools would be needed to accomplish the full characterization and modes extraction, empirical mode decomposition and cyclostationary process analysis represent some of the techniques that will be investigated in the future.

\section{References}

[1] V. C. Chen. "The Micro-Doppler Effect in Radar", Artech House.

[2] C. Clemente, A. Balleri, K. Woodbridge, J. J. Soraghan. "Developments in target micro-Doppler signatures analysis: radar imaging, ultrasound and through-the-wall radar", EURASIP Journal on Advances in Signal Processing, 2013,Springer.

[3] C. Clemente, D. Gaglione, F. K. Coutts, G. Li, J. J. Soraghan. "Model-based sparse recovery method for automatic classification of helicopters", 2015 IEEE Radar Conference (RadarCon). IEEE, 2015. p. 1161-1165.

[4] R. I. A. Harmanny, J. J. M. de Wit, G. PrÃ@cmel Cabic. "Radar Micro-Doppler Feature Extraction Using the Spectrogram and the Cepstrogram", European Radar Conference (EuRAD), 2014 11th, 8-10 Oct. 2014.
[5] A. R. Persico, C. Clemente, D. Gaglione, C. Illoudis, J. Cao, L. Pallotta, A. De Maio, I. Proudler, J. J. Soraghan. "On Model, Algorithms and Experiment for Micro-Doppler based Recognition of Ballistic Targets", IEEE Transactions on Aerospace and Electronic Systems, Feb. 2017.

[6] Y. Kim, S. Choudhury,H. J. Kong. "Application of MicroDoppler Signatures for Estimation of Total Energy Expenditure in Humans for Walking and Running Activities", IEEE Journals \& Magazines, Vol. 4, pp 1560-1569, April 2016.

[7] A. Assmann, A. Izzo and C. Clemente. "Efficient Micro-Doppler based pedestrian activity classification for ADAS systems using Krawtchouk moments", 11th International Conference on Mathematics in Signal Processing (IMA), Dec. 2016.

[8] C. Clemente, L. Pallotta, A. Maio, J. J. Soraghan, A. Farina. "A novel algorithm for radar classification based on Doppler characteristics exploiting pseudo-Zernike polynomials", IEEE Transactions on Aerospace and Electronic Systems, vol. 51, pp. 417-430, Jan. 2015.

[9] W. Klippel. "Scanning Vibrometer C5 Hardware and Software Module of the KLIPPEL R\&D SYSTEM", Klippel GmbH, Dresden, Germany.

[10] W. Klippel, J. Schlechter. "Measurement and Visualization of Loudspeaker Cone Vibration", Audio Engineering Society, October 2006.

[11] W. Klippel. "Loudspeaker Nonlinearities: Causes, Parameters and Symptoms", The Journal of Audio Engineer Socity, Volume 54 Issue 10 pp. 907-939; October 2006, Klippel GmbH, Dresden, Germany.

[12] W. Klippel. "Distributed Mechanical Parameters Describing Vibration and Sound Radiation of Loudspeaker Drive Units", The Journal of Audio Engineer Socity, October 2008, Klippel GmbH, Dresden, Germany.

[13] L. L. Beranek, T. Mellow. "Acoustics: Sound Fields and Transducers", Elsevier Inc.

[14] G. Ni, S. J. Elliott. "Wave Interpretation of Numerical Results for the Vibration in Thin Conical Shells", Institute of Sound and Vibration Research, University of Southampton.

[15] B. P. Bogert, M. J. R. Healy, J. W. Tukey. "The Quefrency Analysis of Time Series for Echoes: Cepstrum, Pseudo Autocovariance, Cross-Cepstrum and Saphe Cracking", Proc. of the Symp. on Time Series Analysis, 1963, chapter 15, pp. 209-243, Wiley, New York. 